\title{
Acute multiple cerebral infarction combined with cerebral microhemorrhage in Polycythemia vera: A case report
}

\author{
NAN WANG ${ }^{1,2^{*}}$, LIYANG LIU $^{1 *}$, XIN JIANG ${ }^{1}$, DAOWEI LI $^{1}$ and XIAOHONG CHEN ${ }^{1}$ \\ ${ }^{1}$ Department of Neurology Inspection, People's Hospital of Liaoning Province, Shenyang, Liaoning 110016; \\ ${ }^{2}$ Department of Neurology, China-Japan Union Hospital of Jilin University, Changchun, Jilin 132000, P.R. China
}

Received October 5, 2018; Accepted May 2, 2019

DOI: $10.3892 /$ etm.2019.7926

\begin{abstract}
Polycythemia vera (PV) is one of the rare causes of cerebrovascular disease, whose common manifestations in the nervous system are cerebral infarction and transient ischemic attack. A number of cases of PV patients with bleeding complicated with subdural hemorrhage or cerebral hemorrhage have been previously reported. However, sometimes PV patient with complicated cerebral hemorrhage and lower extremity venous thrombosis have been admitted to the People's Hospital of Liaoning Province. The present case study reports on a patient with acute multiple cerebral infarction with cerebral micro-hemorrhage associated with PV, who was not treated with anti-thrombosis treatment. After bloodletting treatment and hydroxyurea treatment, the patient's condition was stable and they were discharged. A possible mechanism of infarction is that PV may cause abnormal proliferation of red blood cells, white blood cells and platelets in the circulation, resulting in an increase of blood viscosity and reduction of blood flow velocity. Platelet deficiency may cause abnormal blood coagulation function, which may be the reason for the blood and thrombotic diseases in patients with PV.
\end{abstract}

\section{Introduction}

Polycythemia vera (PV) is a myeloproliferative disease characterized by clonal proliferation of hematopoietic stem cells, leading to abnormal increases in circulating red blood cells, white blood cells and platelets, resulting in an increase of blood viscosity and reduction of blood flow velocity (1). These disorders of hemorheology are all factors of thrombosis. In addition, high blood cell specific capacity may lead to vascular endothelial

Correspondence to: Dr Xiaohong Chen, Department of Neurology Inspection, People's Hospital of Liaoning Province, 33 Wenyi Road, Shenyang, Liaoning 110016, P.R. China

E-mail: cxh_ly@163.com

*Contributed equally

Key words: cerebral, infarction, microhemorrhage, polycythemia vera injury, increasing susceptibility to vascular disease (2-4). The platelet dysfunction in PV patients is mainly manifested as the impairment of aggregation function, which leads to hemostasis disorder, therefore platelet dysfunction is an important factor in bleeding events (5). High hematocrit (HCT) in PV patients can quickly lead to vascular endothelial injury, and the continuous high blood volume may cause the exudation from local blood vessels (3). Dysfunctions in platelet aggregation and the prolongation of activated partial prothrombin time (aPPT) may cause cerebral hemorrhage (6). Several studies have reported that $\mathrm{PV}$ is frequently combined with cerebral infarction or cerebral hemorrhage (7-9). In the current study, the authors reported that one patient with acute cerebral infarction and multiple cerebral microhemorrhage combined with PV, and analyzed imaging features and the pathogenesis.

\section{Case report}

Case presentation. A 60-year-old female patient was admitted to the department of Neurology Inspection, People's Hospital of Liaoning Province (Shenyang, China) on the 13th of November 2017. She complained about sudden impairment of right limb movement for 1 day and slurred speech for $12 \mathrm{~h}$. This patient had been taking anti-psychotic drugs, including chlorpromazine $25 \mathrm{mg} /$ day, alprazolam $0.4 \mathrm{mg} /$ day and trihexyphenidyl $2 \mathrm{mg}$ /day for six months due to schizophrenia. The patient had poor general self-care ability due to mental disorders, who was taken care of by her husband. The patient's husband told the authors that she had been hypertensive for 5 years without any systematic diagnosis and treatment. In recent years, large ecchymosis frequently appeared in the limbs after bumping into a table. At six months previously, the patient was reported to operate slowly, be unresponsive and fall easily, and had therefore been hospitalized for six months in a mental health center, and treated with chlorpromazine $25 \mathrm{mg} /$ day, alprazolam $0.4 \mathrm{mg} /$ day and trihexyphenidyl $2 \mathrm{mg} /$ day, the symptoms were relieved. A complete routine laboratory blood analysis revealed that the patient had no abnormalities, e.g. erythrocytosis, in May 2017. At 1 week prior to admission, the patient developed epistaxis and visited the otolaryngology department to stop the bleeding.

Examination. Physical examination revealed that the blood pressure was 220/120-148-87 $\mathrm{mmHg}$ during hospitalization. 
The patient's consciousness was fluctuating and dominated by fuzzy states (associated with anti-psychotic drugs). In the awake state, the speech was slurred, and the skin of the head, face and palm were dark red. The left nasolabial fold was shallow. The sensation of pain in left facial was lost. The muscle strength was grade 0 in the right limbs, grade 3 in the left upper limb and grade 0 in the lower left limb. The muscular tension did not increase or reduce in the limbs. The patient had a Babinski sign $(\mathrm{L}+, \mathrm{R}+)$ and cervical ankylosis. The lower mandible was three horizontal fingers from the anterior chest.

Laboratory examination revealed that the complete blood count and coagulation were obviously abnormal (Table I). Hemoglobin and HCT were elevated in this patient, and aPPT was prolonged. Through the treatment of bloodletting and Hydroxycarbamide, the patient's HCT reduced a little and the number of white cell returned to normal. At multiple time-points, the blood glucose and glycosylated hemoglobin levels were also abnormal. The maximum blood glucose level reached $19.22 \mathrm{mmol} / \mathrm{l}$. Renal function was abnormal. BUN levels were $13.78 \mathrm{mmol} / \mathrm{l}$, creatinine levels were $155.4 \mu \mathrm{mol} / \mathrm{l}$ (multiple abnormality) and potassium levels were $5.68 \mathrm{mmol} / \mathrm{l}$. Regarding blood lipids, high-density lipoprotein was normal, triglycerides were $2.62 \mathrm{mmol} / 1$, cholesterol was $7.88 \mathrm{mmol} / \mathrm{l}$ and low-density lipoprotein was $5.51 \mathrm{mmol} / \mathrm{l}$. Erythropoietin levels were $3.86 \mathrm{mIU} / \mathrm{ml}$ (5.4-31). Genes were not detected. The patient underwent bone marrow puncture and blood smear examination one week after hospitalization. The results indicated that granulocytes, erythrocytes and megakaryocytes proliferated.

Ultrasound of the liver, gallbladder and spleen revealed fatty liver and splenomegaly (thick diameter, $4.7 \mathrm{~cm}$; long diameter, $11.4 \mathrm{~cm}$ ). Ultrasound of the heart indicated concentric hypertrophy in the left ventricular wall, while no mural thrombosis or obvious valvular disease were present. Ultrasound of the carotid artery revealed no stenosis and occlusive changes had occurred in the extracranial carotid artery, vertebral artery and subclavian artery. On computed tomography of the head, multiple dot-like and flaky low-density changes in the left occipital lobe, the site adjacent to the body of the lateral ventricle, basal ganglia, right frontal lobe and parietal lobe were observed (Fig. 1).

Magnetic resonance images (MRI) of the head were shown in different figures: Fig. 2 was T1 weight imaging, Fig. 3 was T2 weight imaging, Fig. 4 was fluid-attenuated inversion recovery, Fig. 5 was diffusion-weighted image, Fig. 6 was enhanced magnetic resonance imaging and Fig. 7 was susceptibility weighted imaging. Fig. 2 showed the mixed signal intensities, mainly low signal intensity, in the round lesions adjacent to the left body of the lateral ventricle, in line with the changes in the hemorrhagic transformation (type HI-1) post-infarction. Fig. 3 showed multiple intracranial cerebral infarctions, and that round high-signal intensity in the site adjacent to the left body of the lateral ventricle had low signal changes, which was in line with the changes in the hemorrhagic transformation (type HI-1) post infarction. Fig. 4 was fluid-attenuated inversion recovery, which showed the lesions of the right frontal and parietal lobes presented as multiple mixed signals. Fig. 5 was diffusion-weighted image, which showed the acute cerebral infarction, and infarcts with supratentorial, infratentorial and bilateral multiple high-signal intensities. The enhanced magnetic resonance imaging in Fig. 6 had a faint dot-like enhanced signal in the right frontal parietal lobe and the right frontal lobe. Fig. 7 demonstrated multiple dot-like, striped and lumpy low-signal changes.

MRI of the head revealed that the left posterior cerebral artery was occlusive, and that the right anterior cerebral artery and left vertebral artery were thinned. No distinct flow void signal was observed in the conventional sequence, suggesting multiple intracranial microhemorrhage in this area.

Diagnosis. The patient was diagnosed with acute multiple cerebral infarction combined with cerebral microhemorrhage and hemorrhagic transformation. Left posterior cerebral artery occlusion was likely and the right anterior cerebral artery and the left vertebral artery were thin. The patient was also diagnosed with PV and splenomegaly, stage 3 hypertension (very high-risk group), type 2 diabetes and renal insufficiency, abnormal blood lipids, electrolyte disturbance, and abnormally high potassium.

Treatment. After admission, the patient was treated with $30 \mathrm{mg}$ nifedipine for hypertension and intravenously administrated with $60 \mathrm{mg}$ furosemide for the abnormally high potassium. The patient was injected intravenously with $20 \mathrm{ml} 10 \%$ glucose to treat hypoglycemia. Due to renal insufficiency in the compensatory period, no clinical medication was given. Medication was administrated immediately after admission to the hospital. Blood pressure was controlled by intravenous injection of urapidil and sodium nitroprusside. Two or three oral hypertensive medications were combined and administered at the same time, including amlodipine besylate, benidipine, irbesartan and metoprolol. The patient endured the cerebral infarction combined with multiple cerebral microhemorrhage and hemorrhagic transformation, so that no anti-thrombotic drugs were administered. Bloodletting therapy was performed twice, and $380 \mathrm{ml}$ of venous blood was taken out in two sessions 5 days apart. Due to the increasing erythrocyte count and platelet count, and prolonged prothrombin time and activated partial thromboplastin time, stimulation of bone marrow hyperplasia after bloodletting could not be excluded. Thus, the bloodletting therapy was terminated. Hydroxycarbamide was orally administered for 3 days. Conventional blood analysis indicated an obvious improvement. Furthermore, the skin color of the head, face and both hands tended to be more like the normal color than before and stable. The blood pressure and the intracranial condition were stable. At 13 days after admission, the patient was administered hydroxycarbamide (10 mg/kg/day), leading to a reduction in the red blood cell count and inhibition of the hematopoietic ability of the bone marrow, which is a typical effect of hydroxycarbamide treatment. The patient's consciousness changed from fuzzy to awake; the condition was stable and the patient was discharged. The patient was prescribed $30 \mathrm{mg}$ of the anti-hypertensive drug nifedipine sustained release tablets per day after discharge, and the blood pressure was controlled within the normal range. The patient did not receive any anti-coagulant or anti-platelet therapy.

\section{Discussion}

PV is a myeloproliferative neoplasm characterized by clonal erythrocytosis (1). PV is characterized by amplification of 
Table I. Blood routine parameters and indicators of coagulation function.

\begin{tabular}{lcccccccc}
\hline & $\mathrm{RBC}\left(\mathrm{x} 10^{12} / \mathrm{l}\right)$ & $\mathrm{Hb}(\mathrm{g} / \mathrm{l})$ & $\mathrm{HCT}(\%)$ & $\mathrm{WBC}\left(\mathrm{x} 10^{9} / \mathrm{l}\right)$ & $\mathrm{PLT}\left(\mathrm{x} 10^{9} / \mathrm{l}\right)$ & $\mathrm{PT}(\mathrm{sec})$ & $\mathrm{aPPT}(\mathrm{sec})$ & $\mathrm{FIB}(\mathrm{g} / \mathrm{l})$ \\
\cline { 2 - 8 } Normal ranges & $3.8-5.1$ & $115-150$ & $35-45$ & $3.5-9.5$ & $125-350$ & $9-13$ & $21.1-36.5$ & $1.8-3.5$ \\
\hline Day after admission & & & & & & & \\
1 & 7.56 & 231 & 72.7 & 22.18 & 340 & 4.8 & 50.2 & 4.50 \\
2 & 7.17 & 215 & 71.5 & 18.63 & 279 & 14.3 & 44.1 & 4.26 \\
3 & 6.57 & 201 & 66.0 & 22.43 & 301 & 13.6 & 42.4 & 3.99 \\
5 & 7.71 & 231 & 74.3 & 23.26 & 352 & 21.3 & 65.4 & 3.84 \\
$6^{\mathrm{a}}$ & 7.30 & 221 & 68.6 & 24.70 & 349 & 25.0 & 63.5 & 2.49 \\
9 & 7.40 & 223 & 71.0 & 24.59 & 450 & 29.1 & 56.9 & 4.14 \\
$11^{\mathrm{a}}$ & 7.57 & 228 & 72.5 & 19.97 & 480 & 45.8 & 82.2 & 4.14 \\
$16^{\mathrm{b}}$ & 6.92 & 208 & 63.7 & 14.33 & 327 & 16.2 & 39.4 & 5.28 \\
20 & 7.34 & 217 & 70.3 & 7.31 & 265 & 16.7 & 56.9 & 4.63 \\
\hline
\end{tabular}

${ }^{a}$ Re-examination results on day 2 after two courses of bloodletting therapy $(180+200 \mathrm{ml})$; ${ }^{b}$ re-examination results after treatment with hydroxycarbamide $(0.5 \mathrm{~g}$, three times a day, orally, for 3 consecutive days). RBC, red blood cells; Hb, hemoglobin; HCT, hematocrit; WBC, white blood cells; PLT, platelets; PT, prothrombin time; aPPT, activated partial PT; FIB, fibrinogen.
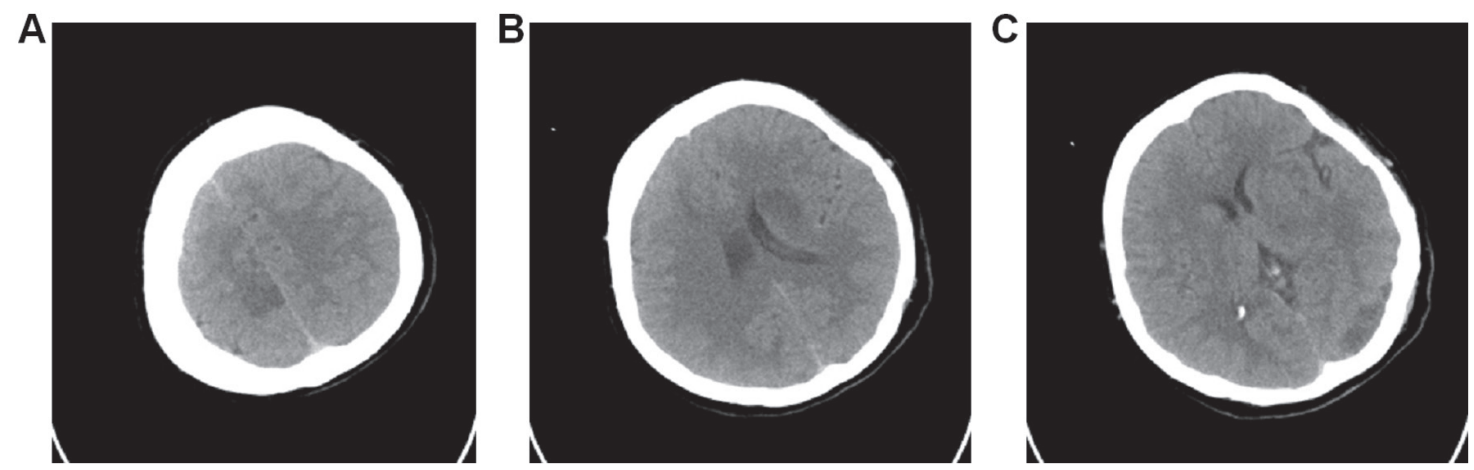

Figure 1. Computed tomograms of the head a day after admission. (A) Multiple dot-like and flaky low-density changes were visible in the left occipital lobe and basal ganglia. The site adjacent to the left body of the lateral ventricle, (B) right frontal lobe and (C) parietal lobe.
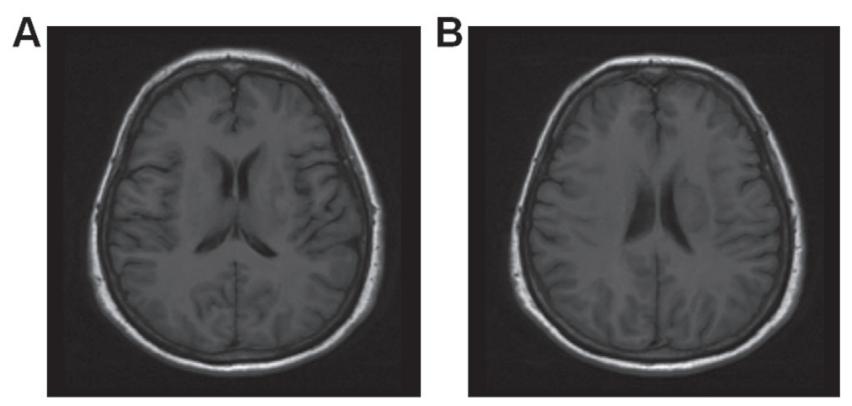

Figure 2. T1-weighted magnetic resonance image 2 days after admission. (A and B; obvious in B) Mixed signal intensities, mainly low signal intensity, in the round lesions adjacent to the left body of the lateral ventricle, (B) in line with the changes in the hemorrhagic transformation (type HI-1) post-infarction.

one or more blood cell lineages, resulting in increased mature blood components in the peripheral blood, including erythrocytosis, leukocytosis and/or thrombocytosis (1). According to the diagnostic criteria set by the World Health Organization in 2016, the diagnosis of PV is mainly based on a comprehensive assessment of clinical and laboratory characteristics (10). Hemoglobin and HCT were elevated in this patient. Bone marrow aspiration and blood smear demonstrated the proliferation of granulocytes, erythrocytes and megakaryocytes, and reduction of serum erythropoietin. Various laboratory and imaging examinations excluded other causes for polycythemia, so the diagnosis of PV was confirmed.

PV may include unique thrombosis and hemorrhage $(5,11)$. The incidence of hemorrhage was reported to be 2-20\% (12-15). Hemorrhage is usually observed in the skin mucosa, and epistaxis, gingival bleeding and gastrointestinal bleeding may also occur (16). Massive epistaxis had occurred in the patient of the present study at one week prior to admission. In recent years, unexplained bruising has frequently occur in the patient, which may be associated with PV. However, there was no abnormality in blood tests performed six months previously, which may indicate an insidious onset of PV. The incidence of thrombotic complications in PV patients was reported to be $12-39 \%(5,12,17)$. Thrombosis is commonly detected in the extremities, mesentery, cerebral blood vessels and coronary arteries. In the patient of the present study, cerebral blood 

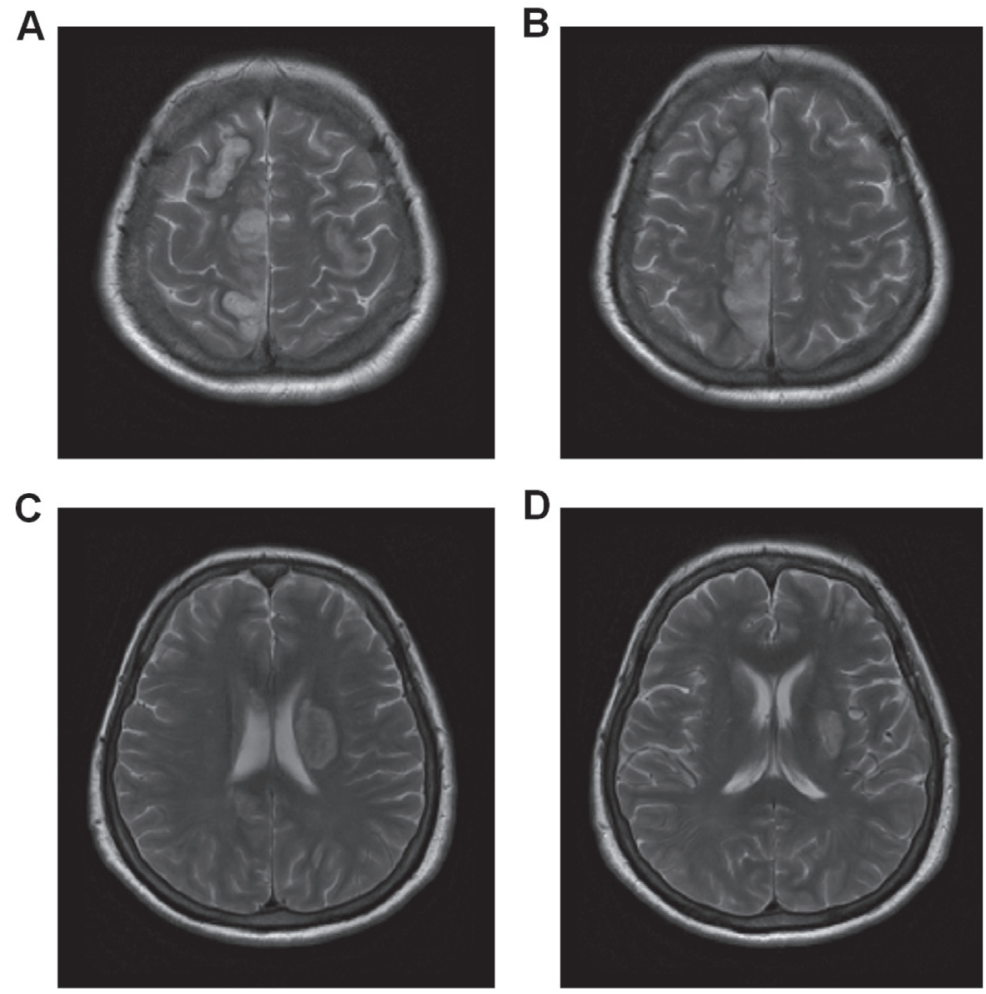

Figure 3. T2-weighted magnetic resonance image 2 days after admission. (A and B) Multiple intracranial cerebral infarction was observed, in which the right frontal parietal lobe lesions exhibited multiple mass-like, discontinuous high-signal intensities and a low-signal ring in the periphery. (A and B) The low-signal ring indicated remote hemorrhage or hemosiderin deposition, mainly in the predominant area of the anterior cerebral artery. (C and D) Round high-signal intensity in the site adjacent to the left body of the lateral ventricle revealed slightly low signal changes, in line with the changes in the hemorrhagic transformation (type HI-1) post-infarction.

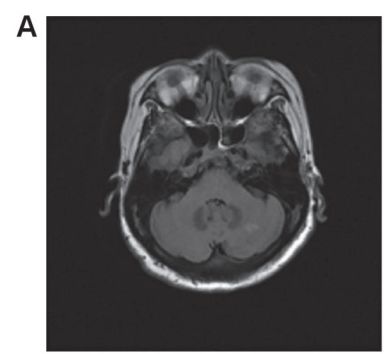

E
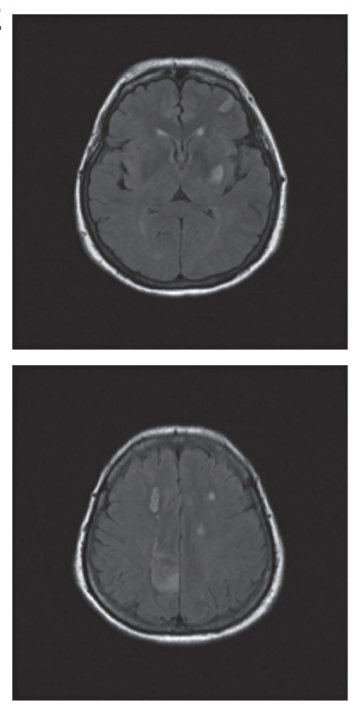
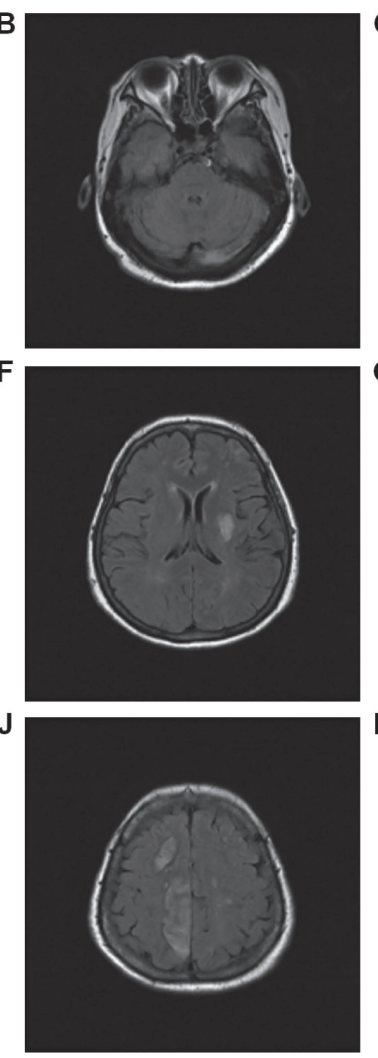

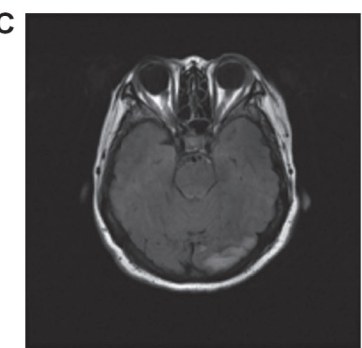

G
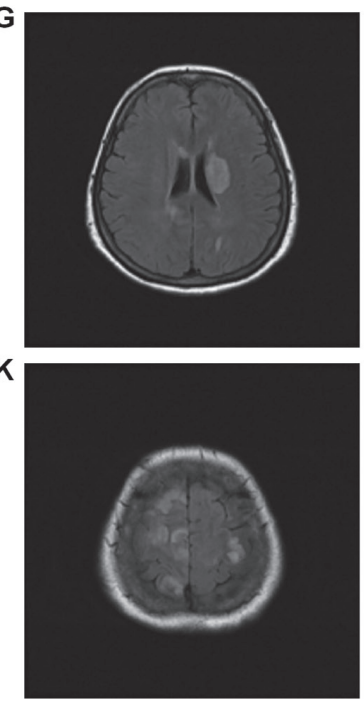

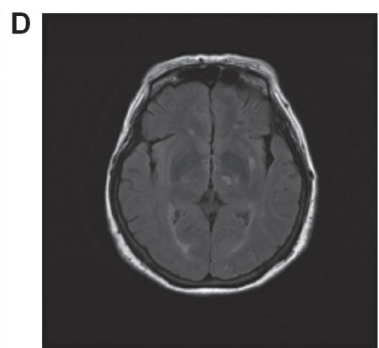

$\mathbf{H}$

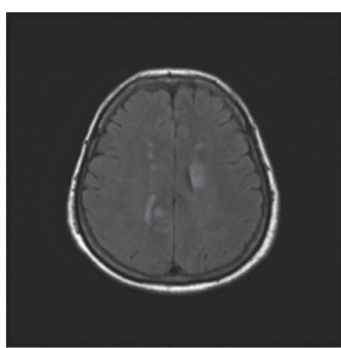

L

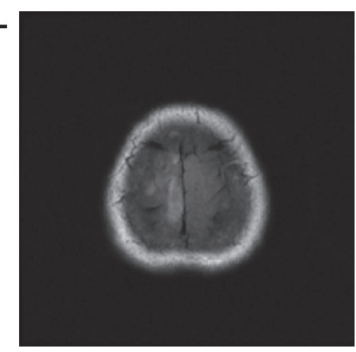

Figure 4. A fluid-attenuated inversion recovery magnetic resonance image sequence 2 days after admission. The sequence was as follows: Multiple, dot-like, striped, and mass-like high-signal lesions in (A) the cerebellar hemisphere, (B and C) the left occipital lobe, (D-F) the thalamus and basal ganglia, (G and $\mathrm{H})$ the site adjacent to the body of the lateral ventricle, (E) the frontal lobe, $(\mathrm{J}$ and $\mathrm{L}$ ) the right frontal lobes, and ( $\mathrm{I}$ and $\mathrm{K})$ the parietal lobes. The lesions of the right frontal and parietal lobes presented as multiple mixed signals. 

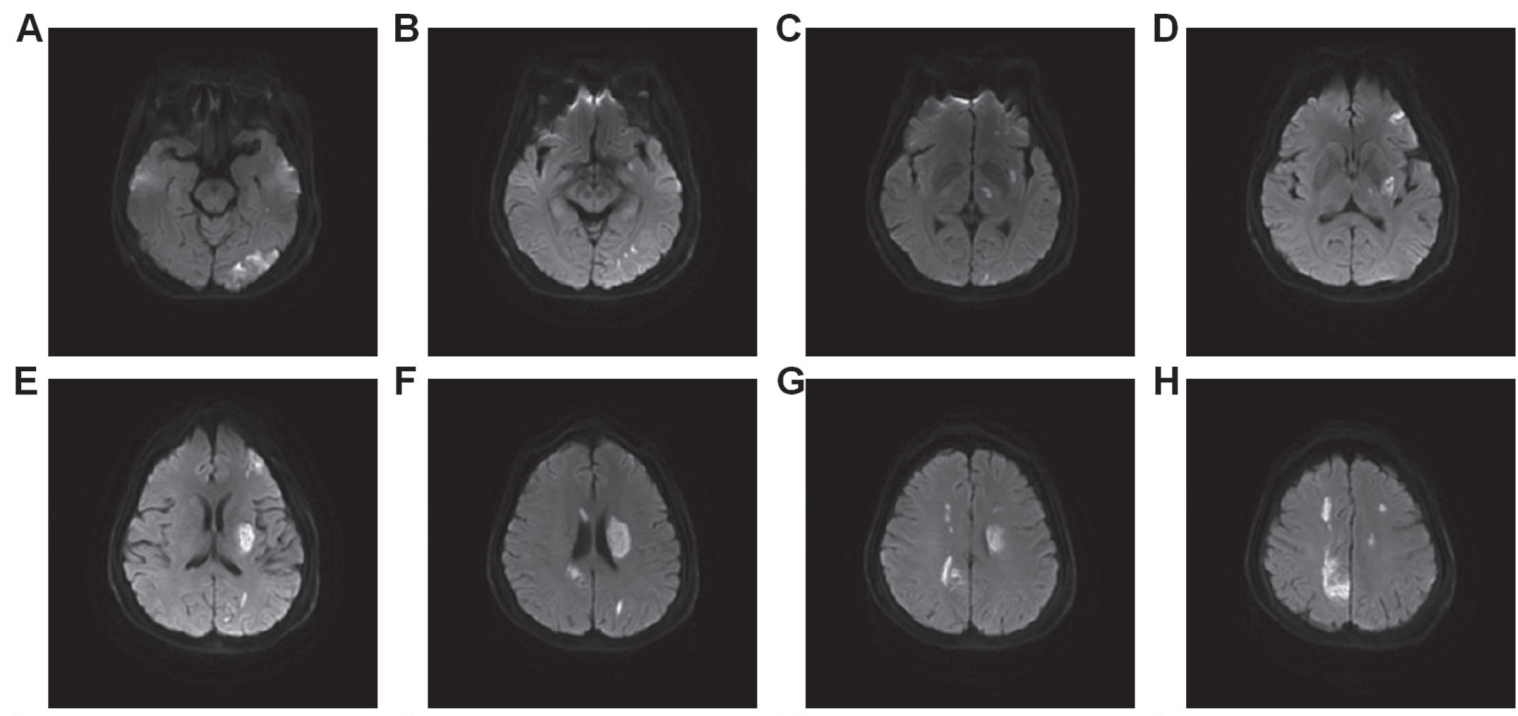

I
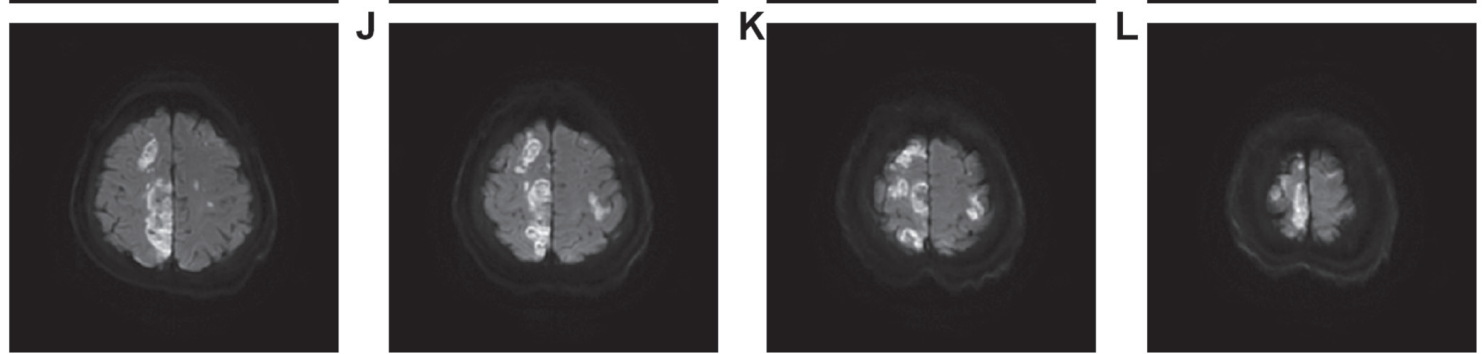

Figure 5. Diffusion-weighted magnetic resonance image of the head 2 days after admission. Acute cerebral infarction, infarcts with supratentorial, infratentorial and bilateral multiple high-signal intensities. (A and B) The lesions of occipital lobe, (C-E) the lesions of thalamus and basal ganglia, (F and G) the lesions of the site adjacent to the body of the lateral ventricle, and (H-L) the lesions of right frontal and parietal lobes.

vessels were mainly involved. According to MRI of the head, this patient was diagnosed with acute multiple cerebral infarction combined with multiple cerebral microhemorrhage and hemorrhagic transformation.

According to a previous study, PV was mainly combined with bilateral multiple cerebral infarction, mainly in the basal ganglia, thalamus, coronaradiate and subcortical lobe, but large-area cerebral infarction was rare (17). In the current study, the patient hemorrhaged in the hypothalamus, base arum and cerebral; the patient also exhibited cerebral microhemorrhage, which has not yet been reported, to the best of our knowledge.

There are numerous mechanisms for ischemic events in PV patients. Leukocytosis and erythrocytosis are risk factors for thrombosis (14-16). Excessive erythrocytosis may significantly increase the HCT and blood viscosity and reduce the cerebral blood flow velocity (15). Theses hemorheologic changes are all thrombophilic factors. Furthermore, chronic excess red blood cells and persistent high HCT may cause vascular endothelial damage, similar to an inflammatory response process that ultimately increases the susceptibility to vascular disease (16). In this case, the erythrocy te HCT and white blood cells of the patient were significantly increased, which are risk factors for thrombosis. An important factor associated with bleeding events in PV patients is abnormal in platelet function, which is also an important cause of clinical manifestations of hemorrhage and ischemia in PV patients. Platelet dysfunction in PV patients is mainly manifested in impaired aggregation function, including reduced reactivity to adrenaline and ADP14, resulting in disorders of hemostasis and prolonged aPPT. High HCT in PV patients easily causes vascular endothelial injury, and a constantly high blood volume causes local oozing of blood vessels, defects in platelet aggregation function and prolonged aPPT, which are all possible causes of the brain microbleeds in the patient of the present study. In addition, platelet dysfunction may also manifest as spontaneous aggregation (17), suggesting a mechanism for regulating platelet activation. This aggravation of hypofunction and spontaneous platelet aggregation frequently occur in the same patient, having a role in disease-associated processes.

The effects of atherosclerosis-associated risk factors on the risk or condition of thromboembolic disease in PV patients are not sufficiently outlined in current guidelines. Hypertension, hyperlipidemia, diabetes and congestive heart failure are risk factors for embolic events in PV patients, and it is suggested that these risk factors should be controlled (18). In the patient of the present study, granulocytes, erythrocytes and megakaryocytes had obviously proliferated, and there were further risk factors for multiple stroke, which increased the chance of occurrence of thrombotic diseases. The blood pressure was high and difficult to control, which was associated with the increased blood volume due to increased blood constituents caused by PV. The pathogenesis of cerebral microhemorrhage and hemorrhagic transformation was considered to be associated with abnormal coagulation function and persistent and obvious hypertension, which further increased the risk of bleeding. 

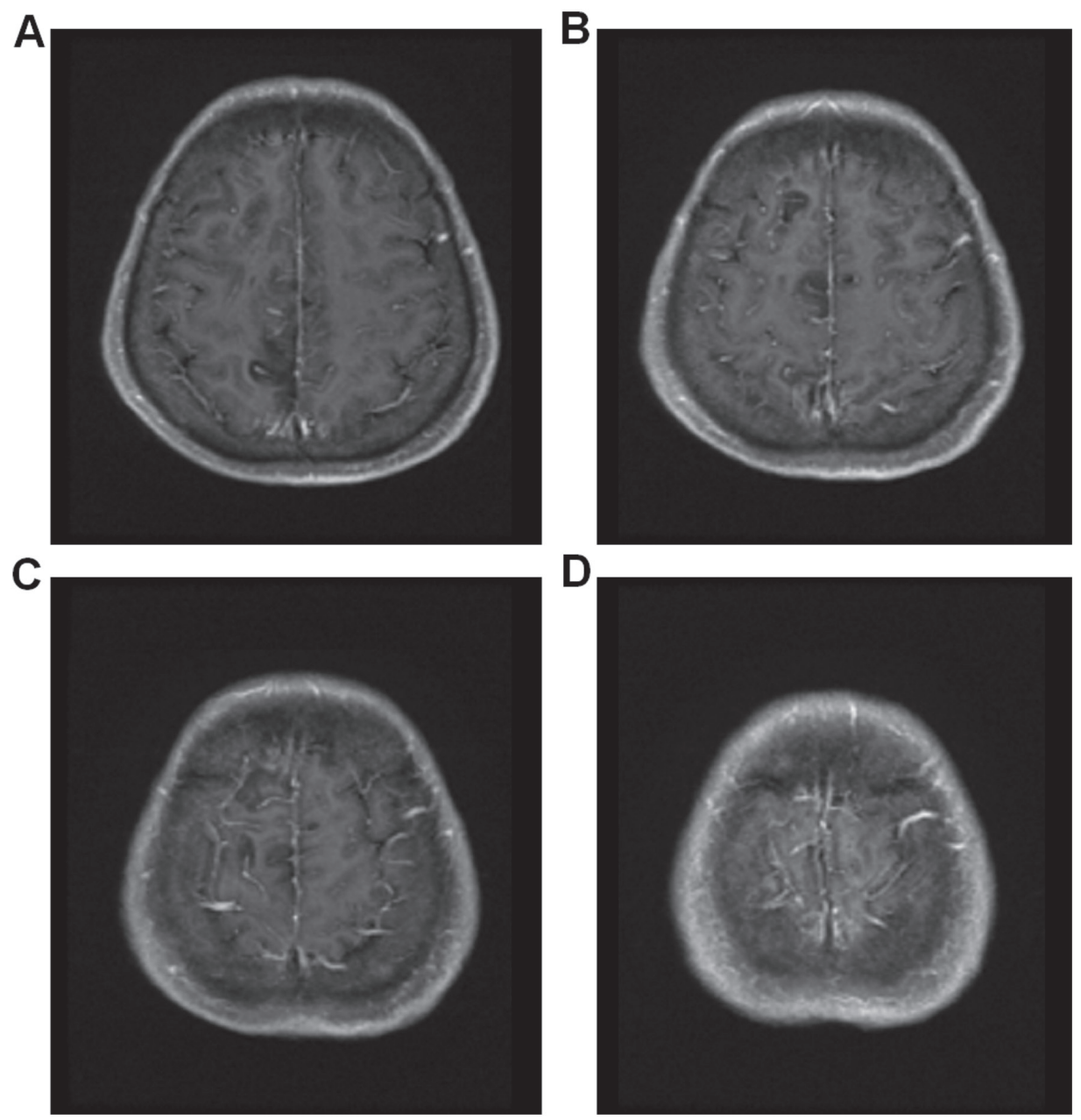

Figure 6. Enhancement magnetic resonance image of the head 2 days after admission. A faint dot-like enhanced signal in (A and B) the right frontal parietal lobe and $(\mathrm{C}$ and $\mathrm{D})$ the right frontal lobe was observed.
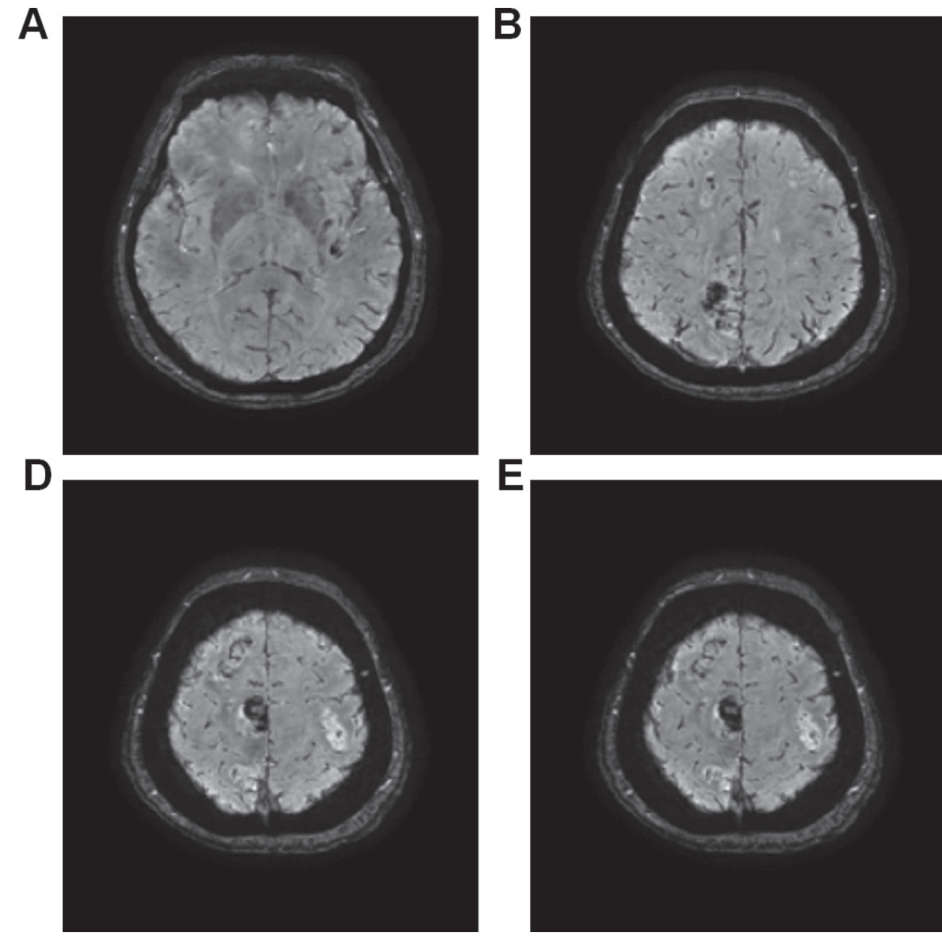

$E$

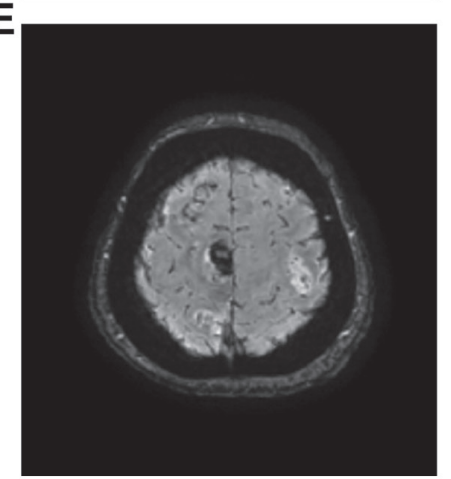

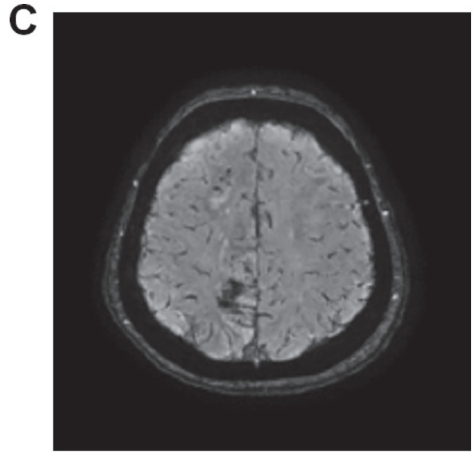

$\mathbf{F}$

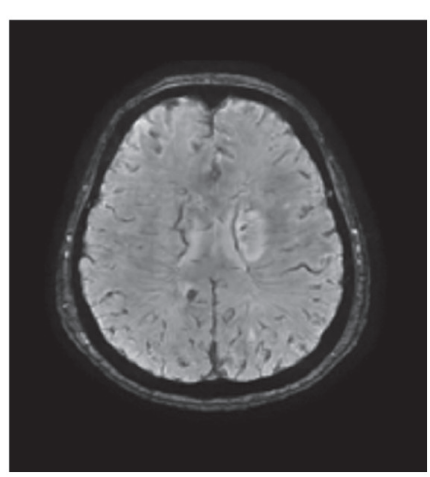

Figure 7. Susceptibility-weighted angiography magnetic resonance image of the head 2 days after admission. (A-C) Multiple dot-like, (D and E) striped and (F) lumpy low-signal changes. 
The patient had no history of heart disease. There was no arrhythmia or heart valve disease, including atrial fibrillation after continuous electrocardiogram (ECG) monitoring, repeated ECG and echocardiography. The presence of multiple infarct foci did not support the diagnosis of cardiogenic brain embolism. However, the patient suffered from multiple intracranial lesions and hemorrhagic transformation, and it was important to identify those. MRI indicated that the case was combined with anterior cerebral artery stenosis, which should be distinguished from atherosclerotic cerebral infarction. However, the infarcts were supratentorial, infratentorial, bilateral and multiple; the infarct focus in the same artery-dominated region was also multiple, which does not conform to the characteristics of atherosclerotic cerebral infarction. Taken together, only cerebral venous system involvement cannot fully explain the mechanism of infarction, but it does not exclude the possibility of simultaneous involvement of the cerebral arteries and cortical veins.

In the present case, the ery throcyte, leukocyte and platelet levels were obviously increased, and the blood fluidity was significantly reduced, leading to the hypercoagulable state of the blood. Although an increase in platelets was obvious, the platelet function was defective, and the PT and the aPPT were prolonged. It was suggested that there is a risk of thrombotic disease as well as a tendency to bleed.

\section{Acknowledgements}

Not applicable.

\section{Funding}

No funding was received.

\section{Availability of data and material}

All data generated or analyzed during the current study are included in this published article.

\section{Authors' contributions}

All the authors analyzed the case and data, and wrote the paper. LL was responsible for the treatment of the patient and collecting patient clinical data. XJ and XHC were in charge of analyzing and interpreting the patient data. DL performed the magnetic resonance examination of brain and provided MRI reports. NW collected patient clinical data and was a major contributor in writing the manuscript. All authors read and approved the final manuscript.

\section{Ethics approval and consent to participate}

The patients signed the informed consent form.

\section{Patient consent for publication}

Written informed consent was obtained from the patient prior to participation in the present case report and the patient consented to publication of images.

\section{Competing interests}

The authors declare that they have no competing interests regarding the publication of this article.

\section{References}

1. Spivak JL: Polycythemia Vera. Curr Treat Options Oncol 19: 12, 2018.

2. Landolfi R, Di Gennaro L, Barbui T, De Stefano V, Finazzi G, Marfisi R, Tognoni G and Marchioli R; European Collaboration on Low-Dose Aspirin in Polycythemia Vera (ECLAP): Leukocytosis as a major thrombotic risk factor in patients with polycythemia vera. Blood 109: 2446-2452, 2007.

3. Lim Y, Lee JO, Kim SH, Kim JW, Kim YJ, Lee KW, Lee JS and Bang SM: Prediction of thrombotic and hemorrhagic events during polycythemia vera or essential thrombocythemia based on leukocyte burden. Thromb Res 135: 846-851, 2015.

4. Marchioli R, Finazzi G, Specchia G, Cacciola R, Cavazzina R, Cilloni D, De Stefano V, Elli E, Iurlo A, Latagliata R, et al: Cardiovascular events and intensity of treatment in polycythemia vera. N Engl J Med 368: 22-33, 2013.

5. Falanga $\mathrm{A}$ and Marchetti M: Thrombotic disease in the myeloproliferative neoplasms. Hematology Am Soc Hematol Educ Program 2012: 571-581, 2012.

6. Zhang H, Sun L, Tang Y, Wang M and Wu J: Effects of Naomaitai Capsule on cerebral blood flow and apoptosis of hippocampus neuron in rats with vascular dementia induced by chronic forebrain ischemia. J Int Neurol Neurosurgery 36: 290-293, 2009.

7. Arboix A, Besses C, Massons J and Titus F: Cerebral infarction as the first manifestation of polycythemia vera. Med Clin (Barc) 101: 398-399, 1993 (In Spanish).

8. Yazdi R and Côté C: Watershed infarction in a case of polycythemia vera. Clin Nucl Med 11: 665-666, 1986.

9. Xin $\mathrm{CH}, \mathrm{Xu} J Q$, Sui JR and Wang XL: Analysis on 71 patients with polycythemia vera. Zhongguo Shi Yan Xue Ye Xue Za Zhi 20: 667-670, 2012.

10. Zou JM, Pan ZJ and Wang SL: Pharmacodynamic and toxicologic research on nao mai kang capsule. China J Traditional Chin Med Pharm 18: 408-413, 2003.

11. Zhang HN, Sun L, Tang YY, Wang MY and Wu J: Effects of Naomaitai Capsule on cerebral blood flow and apoptosis of hippocampus neuron in rats with vascular dementia induced by chronic forebrain ischemia. J Int Neurol Neurosurgery 36: 290-293, 2009.

12. Elliott MA and Tefferi A: Thrombosis and haemorrhage in polycythaemia vera and essential thrombocythaemia. $\mathrm{Br}$ J Haematol 128: 275-290, 2005.

13. Kander EM, Raza S, Zhou Z, Gao J, Zakarija A, McMahon BJ and Stein BL: Bleeding complications in BCR-ABL negative myeloproliferative neoplasms: Prevalence, type, and risk factors in a single-center cohort. Int J Hematol 102: 587-593, 2015.

14. Barbui T, Carobbio A, Rumi E, Finazzi G, Gisslinger H, Rodeghiero F, Randi ML, Rambaldi A, Gisslinger B, Pieri L, et al: In contemporary patients with polycythemia vera, rates of thrombosis and risk factors delineate a new clinical epidemiology. Blood 124: 3021-3023, 2014.

15. Marchioli R, Finazzi G, Landolfi R, Kutti J, Gisslinger H, Patrono C, Marilus R, Villegas A, Tognoni G and Barbui T: Vascular and neoplastic risk in a large cohort of patients with polycythemia vera. J Clin Oncol 23: 2224-2232, 2005.

16. Landolfi R, Cipriani MC and Novarese L: Thrombosis and bleeding in polycythemia vera and essential thrombocythemia: Pathogenetic mechanisms and prevention. Best Pract Res Clin Haematol 19: 617-633, 2006.

17. Tefferi A: Polycythemia vera and essential thrombocythemia: 2012 update on diagnosis, risk stratification, and management. Am J Hematol 87: 285-293, 2012.

18. Cerquozzi S, Barraco D, Lasho T, Finke C, Hanson CA, Ketterling RP, Pardanani A, Gangat N and Tefferi A: Risk factors for arterial versus venous thrombosis in polycythemia vera: A single center experience in 587 patients. Blood Cancer J 7: 662, 2017. 